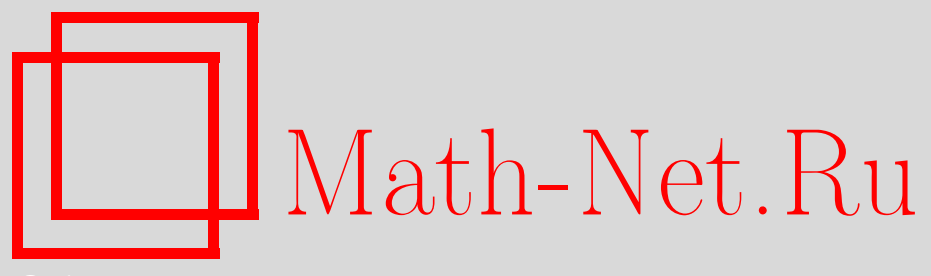

В. В. Подольский, А. А. Шерстов, Уменьшение на единицу степени многочлена с заданной знаковой функцией может экспоненциально увеличить его вес и длину, УМH, 2009, том 64, выпуск 5, 179-180

DOI: https://doi.org/10.4213/rm9303

Использование Общероссийского математического портала Math-Net.Ru подразумевает, что вы прочитали и согласны с пользовательским соглашением http://www . mathnet.ru/rus/agreement

Параметры загрузки:

IP: 107.22 .136 .117

26 апреля 2023 г., 17:02:04

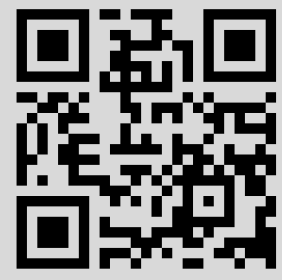




\section{Уменьшение на единицу степени многочлена с заданной знаковой функцией может экспоненциально увеличить его вес и длину}

\section{В. В. Подольский, А. А. Шерстов}

Булева функция $f:\{-1,+1\}^{n} \rightarrow\{-1,+1\}$ называется знаковой функцией целочисленного многочлена $p\left(x_{1}, \ldots, x_{n}\right)$, если $f\left(x_{1}, \ldots, x_{n}\right)=\operatorname{sgn} p\left(x_{1}, \ldots, x_{n}\right)$, где под функцией $\operatorname{sgn}$ мы понимаем знаковую функцию: $\operatorname{sgn} t=1$, если $t>0, \operatorname{sgn} t=0$, если $t=0$, и $\operatorname{sgn} t=-1$, если $t<0$. При этом многочлен $p\left(x_{1}, \ldots, x_{n}\right)$ называется пороговым элементом для булевой функции $f$. Весом порогового элемента называется сумма абсолютных значений его коэффициентов, а его длиной называется количество одночленов в нем. Пороговой степенъю функции $f$ называется наименьшая степень порогового элемента для $f$. Через $W(f, d)$ будем обозначать наименьший вес порогового элемента степени $d$ для функции $f$. Через $L(f, d)$ будем обозначать наименьшую длину порогового элемента степени $d$ для функции $f$. Наконец, через $W^{*}(f, d)$ будем обозначать наименьшее $k$, для которого существуют функции $f_{i}:\{-1,+1\}^{n} \rightarrow$ $\{-1,+1\}, i=1, \ldots, k$, зависящие от не более чем $d$ своих переменных и такие, что $f\left(x_{1}, \ldots, x_{n}\right)=\operatorname{sgn}\left(\sum_{i=1}^{k} f_{i}\left(x_{1}, \ldots, x_{n}\right)\right)$ для всех $x_{1}, \ldots, x_{n} \in\{-1,+1\}$.

Основной вопрос, которым мы задаемся, звучит следующим образом: насколько сильно могут изменяться величины $W(f, d)$ и $L(f, d)$ при небольших изменениях $d$ ? Во всех предыдущих работах величины $W(f, d)$ и $W(f, d+1)$ были полиномиально связаны для всех $d$ (см., например, [1]-[3]). То же самое верно и для величины $L(f, d)$. Наш основной результат - построение для всякого данного $d$ булевой функции $f$ с экспоненциальным разрывом между $W(f, d)$ и $W(f, d+1)$.

Теорема 1. Для всякого $d=1, \ldots, n-1$ существует (явно заданная) функиия $f:\{-1,+1\}^{n} \rightarrow\{-1,+1\}$ такая, что

$$
W(f, d)=\exp \{\Theta(n)\} \quad u \quad W(f, d+1)=O\left(n^{2}\right) .
$$

Другими словами, мы покажем, что необходимый для вычисления заданной функции вес пороговых элементов может сильно измениться в ответ на небольшие изменения степени пороговых элементов.

Кроме того, мы доказываем следующий более сильный результат, относящийся к булевым схемам из функций голосования. Функцией голосования называется знаковая функция порогового элемента степени 1 с коэффициентами \pm 1 . Полезно иметь в виду, что знаковая функция порогового элемента с весом $W$ - это функция голосования от $W$ функций логического сложения $\oplus$ (и наоборот).

Теорема 2. Для всякого $d=1, \ldots, n-1$ существует (явно заданная) функиия $f:\{-1,+1\}^{n} \rightarrow\{-1,+1\}$ такая, что:

1) всякая функиия голосования, примененная $к$ произвольным элементам входной степени не выше $d$ и вычисляющая $f$, должна применяться к не менее чем $\exp \{\Omega(n)\}$ элементам, что также является достаточным;

2) $f$ вычислима функиией голосования от $O\left(n^{2}\right)$ функций логического сложения $\oplus$ входной степени не выше $d+1$, а также функиией голосования от $O\left(n^{2} d\right)$ функиий голосования входной степени не выше $d+1$.

Первый автор поддержан РФФИ (грант № 09-01-00709-а) и программой “Ведущие научные школы" (грант НШ-845.2008.1); второй автор поддержан Национальным научным фондом США (гранты CCF-0728536 и "Career Award" на имя профессора А. Р. Клайванса). 
Заметим, что первая часть теоремы означает, что $W^{*}(f, d)=\exp \{\Theta(n)\}$.

Кроме того, мы доказываем результат, аналогичный теореме 1 , для пороговой длины.

Теорема 3. Для всех $n$ и всякого $d=1, \ldots, n-1$ существует (явно заданная) функиия $f:\{-1,+1\}^{n} \rightarrow\{-1,+1\}$ такая, что

$$
2^{(d-1) / 2} \leqslant L(f, d) \leqslant 2^{d+1}
$$

$u$

$$
L(f, d+1) \leqslant d+3 .
$$

Оценка $L(f, d)$ в этом результате близка к оптимальной, так как существует не более $(n+1)^{d}$ одночленов степени не выше $d$ и, следовательно, длина всякого порогового элемента степени не выше $d$ не превышает $(n+1)^{d}$.

Доказательство теорем 1 и 2 использует совершенно разные методы для различных значений $d$. Более точно, мы доказываем следующие две промежуточные теоремы.

Теорема 4. Для любых $n$ и $d=1, \ldots, n-1$ существует (явно заданная) функиия $f:\{-1,+1\}^{n} \rightarrow\{-1,+1\}$ такал, что

$$
W(f, d)=\Theta\left((n-d) 2^{(n-d) / 2}\right)
$$

$u$

$$
W(f, d+1)=O\left((n-d)^{2}\right) .
$$

Теорема 5. Для всякого $d=1, \ldots, n-1$ существует (явно заданная) функиия $f:\{-1,+1\}^{n} \rightarrow\{-1,+1\}$ такая, что

$$
W^{*}(f, d)=W(f, d)=2 \cdot 2^{d}-1
$$

$u$

$$
W^{*}(f, d+1) \leqslant W(f, d+1) \leqslant 2 d+3 .
$$

Теорема 1 при $d<n / 2$ непосредственно следует из теоремы 4 , а при $d \geqslant n / 2-$ из теоремы 5 . С помощью более сложного анализа из теорем 4 и 5 выводится и теорема 2 .

Авторы благодарны Н. К. Верещагину за полезные обсуждения на тему пороговых элементов.

\section{Список литературы}

[1] M. Goldmann, J. Håstad, A. A. Razborov, Comput. Complexity, 2:4 (1992), 277-300. [2] R. Beigel, Comput. Complexity, 4:4 (1994), 339-349. [3] V. V. Podolskii, Computer sciencetheory and applications, Lecture Notes in Comput. Sci., 5010, Springer, Berlin, 2008, 261-272.

В. В. Подольский (V. V. Podolskii)

Московский государственный университет им. М. В. Ломоносова

E-mail: podolskii@lpcs.math.msu.su

\section{А. А. Шерстов (А. A. Sherstov)}

University of Texas in Austin

E-mail: sherstov@cs.utexas.edu
Представлено В. М. Бухштабером Принято редколлегией 18.06.2009 